\title{
Nach den Polikliniken die Agrokliniken!
}

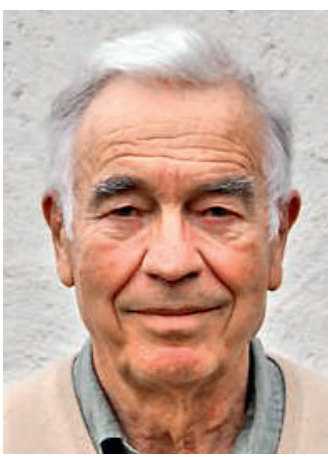

Hans Stalder
Die medizinischen Polikliniken - ihr Name leitet sich vom griechischen Wort $\pi$ ó $\iota_{\iota}$ (Stadt) ab - entstanden gegen Ende des 19. Jahrhunderts in den Schweizer Grossstädten, um die Pflege für mittellose Personen, die keinen Zugang zum Gesundheitswesen hatten, zu sichern. In Genf wurde die Poliklinik im Jahr 1876 gleichzeitig mit der medizinischen Fakultät gegründet. «La policlinique n'est pas autre chose que l'assistance publique sous forme de consultations et de visites à domicile, utilisées au point de vue de l'enseignement. Des élèves déjà avancés, sous le contrôle d'un professeur et de ses assistants, soignent et visitent les malades indigènes»: So wurde die Aufgabe der Poliklinik im Gründungsbericht der medizinischen Fakultät beschrieben

\section{Wäre es nicht genial, zwei hehre Aufgaben des Staates miteinander zu verknüpfen?}

War es nicht genial, zwei hehre Aufgaben des Staates - Solidarität mit jenen, die keinen Zugang zum Gesundheitssystem hatten (lange vor der Einführung der obligatorischen Krankenpflegeversicherung) und akademische Tätigkeit miteinander $\mathrm{zu}$ verknüpfen? Die Polikliniken wurden privilegierte Stätten der ambulanten medizinischen Forschung und der Verwirklichung der sozialen Komponente der Medizin. Sie spielten eine wichtige Rolle in der Entwicklung verschiedener Disziplinen wie Phthisiologie, Vakzinologie oder Tropenmedizin und inspirierten in Genf Spezialgebiete wie Geriatrie, Angiologie, klinische Pharmakologie und Rheumatologie. Die Schweizer Polikliniken haben eine ruhmreiche Vergangenheit mit grossen Klinikleitern wie Hegglin und Siegenthaler in Zürich, Gsell in Basel, Martin und Fabre in Genf oder Jecquier-Doge in Lausanne. Nach der Einführung der obligatorischen Krankenpflegeversicherung wurde die Rolle der Polikliniken als Klinik für die Stadt in Frage gestellt. Mit dem Auftreten einer neuen Armut - man denke an Migranten, Menschen ohne festen Wohnsitz und «Sans-Papiers» oder junge Drogenabhängige - scheint die Existenz von Polikliniken, so wie sie vor allem noch in der Westschweiz zu finden sind, auch heute noch ebenso gerechtfertigt wie früher. Hier können Studierende und künftige Praktiker ambulante Medizin «unter Anleitung eines Professors und seiner Assistenten» erfahren, den Umgang mit Notfällen lernen, Patienten langfristig begleiten und sogar auch Hausbesuche machen.
In letzter Zeit wird immer klarer, dass es eine neue Risikobevölkerung gibt, die keinen Zugang zum Gesundheitswesen hat. Es sind nicht mehr die Armen der Stadt, sondern Menschen auf dem Land, wo es immer weniger Hausärzte gibt. Ihre Grundversorgung ist gefährdet. Könnte da nicht eine ähnliche Institution für diese Risikobevölkerung geschaffen werden, wie dies vor nahezu einem Jahrhundert für die mittellose Stadtbevölkerung getan wurde? Eine Anbindung an die medizinischen Fakultäten hätte einen doppelten Vorteil: Zum einen wäre damit für eine gefährdete Bevölkerungsgruppe der Zugang zum Gesundheitswesen gewährleistet und zum anderen könnten damit Aus- und Weiterbildungsplätze für junge Mediziner geschaffen werden. Heutzutage, wo von den Fakultäten verlangt wird, mehr Studierende aufzunehmen, fehlen klinische Ausbildungsplätze. Seit langem steht die in den Spitälern gängige Ausbildungspraxis als zu technisch und zu spezialisiert in der Kritik. Sie entspricht nicht mehr den Anforderungen der praktischen Medizin. Mit der Einführung der Fallpauschalen, die zu einer Verkürzung der Spitalaufenthalte tendiert und damit keinen Raum mehr für praktische Ausbildung am Krankenbett lässt, ist diese Ausbildungspraxis noch fraglicher geworden. Dazu kommt, dass ihre Finanzierung nicht mehr gesichert ist. Die Begleitung von Patienten mit Langzeiterkrankungen eine der grössten Herausforderungen unserer Zeit ist so jedenfalls nicht mehr möglich. Daher wird der Verlagerung der klinischen Weiterbildung auf den ambulanten Sektor eine herausragende Rolle zukommen.

\section{Hier könnten Studierende und künftige Allgemeinmediziner in ambulanter Medizin aus- gebildet werden.}

Die entsprechenden Risikogebiete müssen von den gesundheitspolitischen Entscheidungsträgern definiert werden. Die Verbindung zu den medizinischen Fakultäten könnte über die universitären Institute für Hausarztmedizin gewährleistet werden. Somit hätten wir universitäre Agrokliniken im Sinne der universitären Polikliniken und könnten die medizinische Versorgung einer gefährdeten Bevölkerungsgruppe und mit einer guten medizinischen Aus- und Weiterbildung sicherstellen!

Hans Stalder* 